\title{
Ultrafast dynamics through conical intersections and intramolecular vibrational energy redistribution in styrene
}

\author{
A. D. G. Nunn ${ }^{1}$, R. S. Minns ${ }^{1}$, R. Spesyvtsev ${ }^{1}$, M.J. Bearpark², M. A. Robb², \\ H. H. Fielding ${ }^{1 *}$ \\ ${ }^{1}$ Department of Chemistry, University College London, 20 Gordon Street, \\ London WC1H OAJ, UK \\ ${ }^{2}$ Department of Chemistry, Imperial College London, South Kensington, \\ London SW7 2AZ, UK
}

\begin{abstract}
We report a femtosecond time-resolved photoelectron spectroscopy (TRPES) investigation of internal conversion in the first two excited singlet electronic states of styrene. We find that radiationless decay through an $\mathrm{S}_{1} / \mathrm{S}_{0}$ conical intersection occurs on a timescale of $\sim 4 \mathrm{ps}$ following direct excitation to $S_{1}$ with $0.6 \mathrm{eV}$ excess energy, but that the same process is significantly slower $(\sim 20 \mathrm{ps})$ if it follows internal conversion from $S_{2}$ to $S_{1}$ after excitation to $S_{2}$ with $0.3 \mathrm{eV}$ excess energy $(0.9 \mathrm{eV}$ excess energy in $\mathrm{S}_{1}$ ).
\end{abstract}

*Email: h.h.fielding@ucl.ac.uk 


\section{Introduction}

Ultrafast relaxation of electronic excited states through conical intersections plays a significant role in many photochemical and photobiological processes. There are many examples of photochemical isomerisations about $\mathrm{C}=\mathrm{C}$ double bonds that have been shown to involve conical intersections, ${ }^{1}$ and styrene has been studied as a prototype for these processes, despite the fact that the two unsubstituted styrene isomers are equivalent. Styrene can also be considered as a substituted benzene, and therefore the electronic relaxation pathways can be expected to have similarities with those of benzene.

The gas-phase ultraviolet (UV) absorption spectrum of styrene in the region up to around $7 \mathrm{eV}$ consists of three main bands. ${ }^{2}$ Historically, there was some debate over the electronic origin of the second band, with most early studies assuming that it arose from excitation to both $\mathrm{S}_{2}$ and $\mathrm{S}_{3} ;{ }^{3-4}$ however, it is now generally accepted that the first two bands arise from excitation to the lowest two singlet electronic states, $\mathrm{S}_{1}$ and $\mathrm{S}_{2}$, and result from excitations within the valence $\pi-\pi^{*}$ molecular orbitals. ${ }^{5-6}$ The zeropoints of the $S_{1}$ and $S_{2}$ states have been determined spectroscopically to be $4.31 \mathrm{eV}^{7}$ and $4.88 \mathrm{eV},{ }^{8}$ respectively.

Philis et al., ${ }^{2,9}$ observed the 3p Rydberg states of styrene at $6.259 \mathrm{eV}, 6.456 \mathrm{eV}$ and $6.529 \mathrm{eV}$ by resonance-enhanced multiphoton ionisation (REMPI), assigned them $3 \mathrm{p}_{y}$, $3 \mathrm{p}_{x}$ and $3 \mathrm{p}_{z}$ by comparison with CASPT 2 calculations, ${ }^{5}$ and determined lower limits for their lifetimes of $91 \mathrm{fs}, 38 \mathrm{fs}$ and 165 fs from linewidth measurements. The absorption coefficients of these Rydberg states are small and their involvement in the photochemistry of styrene has not been reported as significant.

Energy barriers to decay on the $S_{1}$ surface can be estimated from vibronic spectroscopy experiments and for isolated styrene and $\beta$-methylstyrene have been found to be at least $3000 \mathrm{~cm}^{-1}$ above the $S_{1}$ origin. ${ }^{10-12}$ Phosphorescence has never been detected from photoexcited styrene; however, many studies have suggested that photochemical cis-trans isomerisation mechanisms for styrene proceed via triplet states. $^{11,13-15}$

Recent theoretical studies have investigated the decay pathway of photoexcited $\mathrm{S}_{2}$ styrene in some detail. According to Amatatsu, ${ }^{16}$ after passing through the $S_{2} / S_{1}$ conical intersection $(\mathrm{CI})$ with a twisted quinoid geometry, population can either relax directly to the $S_{1}$ equilibrium structure or it can pass to a nearby transition state from where it can then relax to the $S_{1}$ equilibrium geometry and/or proceed on to an $S_{1} / S_{0}$ conical intersection. With insufficient vibrational excess energy, the only possible singlet decay mechanism from the $S_{1}$ equilibrium geometry is fluorescence, which is 
known to have a nanosecond timescale. The only ultrafast decay pathway from $S_{1}$ is to $\mathrm{S}_{0}$ directly via a conical intersection, requiring extra vibrational energy. Bearpark, Robb and coworkers have calculated two different $\mathrm{S}_{1} / \mathrm{S}_{0} \mathrm{CI}$ geometries that could be important to $S_{1}$ decay ${ }^{13}$ and others have also been reported. ${ }^{17}$

In a systematic study of the decay dynamics of a number of substituted benzenes, Stolow and coworkers reported 52 fs $\mathrm{S}_{2} / \mathrm{S}_{1}$ decay followed by 88 ps $\mathrm{S}_{1} / \mathrm{S}_{0}$ decay in styrene excited at the $S_{2}$ origin. ${ }^{18}$ The motivation for our work was to investigate the decay dynamics of photoexcited styrene in more detail.

Experiments measuring the integrated ionisation cross-section as a function of pumpprobe delay cannot differentiate between internal conversion (IC), intersystem crossing (ISC) or the possibility of either of these photophysical processes occurring in combination with photochemistry; particularly isomerisation in this case. The key to developing a detailed picture of the excited state dynamics in a prototypical system such as styrene, and to developing a detailed understanding of the excited state dynamics of any molecule, is to measure the electronic and nuclear composition of the total wave function of the molecule as it evolves. In practice, because the electronic and nuclear motions are strongly coupled, this is not trivial; however, time-resolved photoelectron spectroscopy (TRPES) has emerged as an extremely valuable tool for following excited state non-adiabatic processes in molecules. ${ }^{19-22}$ First, an ultrafast laser pulse (pump) prepares the molecule in an electronically and vibrationally excited non-stationary state, and then, a subsequent ultrafast laser pulse (probe) ionises the molecule and projects the evolving molecular wave function onto the molecular ionisation continuum. By measuring the resulting photoelectron spectrum as a function of pump-probe delay, one can gain some insight into the electronic and geometric changes along the reaction coordinate. TRPES has been employed to monitor wave packet dynamics in simple diatomic molecules, ${ }^{23}$ to follow IC in polyenes, ${ }^{24}$ photodissociation, ${ }^{25-27}$ intramolecular vibrational energy redistribution $(\mathrm{IVR})^{28}$ and ISC. ${ }^{26,29-31}$ However, in molecules in which there is a high density of electronically excited states correlating to the same electronic state of the cation, decomposing the evolving wavefunction into its constituent parts is challenging, and requires the support of theory.

In this paper, we present femtosecond time-resolved photoelectron spectra of styrene collected using different pump wavelengths. The data have been analysed using an ionisation correlation analysis based on time-dependent density functional theory (TDDFT) calculations, and provides direct experimental evidence for decay via different singlet state photophysical pathways in styrene after excitation to $S_{1}$ and $S_{2}$, as suggested by earlier calculations. ${ }^{13}, 16$ 


\section{Theory}

The basic principles of our investigation of the excited state dynamics of styrene are illustrated schematically in figure 1. Immediately after excitation by the pump pulse, the total excited state wavefunction is a linear combination of vibronic wavefunctions, $\psi_{i}(q) e^{-i E t / \hbar}$, integrated over the envelope of the electric field, $\varepsilon(E)$ : $\Psi(t)=\int d E \varepsilon(E) \sum_{i, q} c_{i}^{q}(t) \psi_{i}(q) \exp (-i E t / \hbar)$, where $i$ labels the $S_{n}$ electronic states, $q$ represents the vibrational modes and $c_{i}^{q}(t)$ are the time-dependent amplitudes. The photoelectron spectrum of each of these electronic states can, in principle, be distinguished because they have different overlap integrals between their vibronic wavefunctions and those of the cation; however, the bands are strongly overlapping and not trivial to disentangle (see top right inset in figure 1).

In order to unravel the photoelectron spectrum it is necessary to identify which regions of the photoionisation continuum are associated with ionisation from the different $S_{n}$ electronic states, i.e. we need to determine vibronic transition propensities between styrene and the styrene cation. To a first approximation, we can deduce the ionisation correlations at the Franck-Condon geometry and treat the electronic and vibrational components of the wavefunctions separately. For the vibrational component, if we assume that the vibrational quantum number is approximately preserved during photoionisation, we expect the photon energy threshold for ionisation out of $S_{3}$ to be lower than that from $S_{2}$, which in turn is lower than that for $\mathrm{S}_{1}$ (see figure 1). For the electronic component, we need to establish correlations between the electronic states of the neutral molecule and the electronic states of the cation.

To be able to determine the ionisation correlations at the Franck-Condon geometry, we have carried out quantum chemistry calculations for the electronically excited states of neutral styrene and its cation. The geometry of the ground state of neutral styrene was optimized at the DFT B3LYP/6-31G* level. The optimised geometry is planar, all the in-plane angles are approximately $120^{\circ}$, the $\mathrm{CH}$ distances are $1.09 \AA$, the $\mathrm{CC}$ bond lengths in the benzene ring are $1.40 \AA$, the ethylene $\mathrm{CC}$ bond is $1.34 \AA$ and the CC bond joining the benzene ring to ethylene is $1.47 \AA$. This geometry was used for all other calculations.

Orbitals for the ground state wavefunctions of styrene and the styrene cation (calculated at the DFT HCTH/6-311+G* level) are the reference orbitals for the TDDFT HCTH/6-311+G* excited state calculations ${ }^{32-33}$ and are presented in figure 2. The resulting configurations and weights of the excited state wavefunctions for 
styrene and the styrene cation are summarized in tables 1(a) and (b). ${ }^{*}$ Since our focus here is on experimental assignment, we have used experimental excitation energies instead of those calculated at this level of theory where possible. The TDDFT theory is used here to calculate the electronic state configuration and the correlations between the excited states of the neutral molecule and the cation as described below. The asymptotically corrected HCTH functional ${ }^{32-33}$ was chosen as it was found to give reliable excitation energies for benzene. ${ }^{34}$

\begin{tabular}{|c|c|c|c|c|}
\hline (a) & Energy $(\mathrm{eV})$ & Excitation configuration from $\mathrm{S}_{0}$ & Coefficient & Weight \\
\hline \multirow[t]{3}{*}{$\mathrm{S}_{1}$} & $4.31^{7}(4.47)$ & HOMO- $1 \rightarrow$ LUMO & 0.48 & 0.45 \\
\hline & & $\mathrm{HOMO} \rightarrow$ LUMO & 0.23 & 0.10 \\
\hline & & $\mathrm{HOMO} \rightarrow \mathrm{LUMO}+1$ & -0.47 & 0.44 \\
\hline \multirow[t]{2}{*}{$\mathrm{S}_{2}$} & $4.88^{8}(4.67)$ & HOMO-1 $\rightarrow$ LUMO & -0.26 & 0.13 \\
\hline & & $\mathrm{HOMO} \rightarrow \mathrm{LUMO}$ & 0.64 & 0.82 \\
\hline \multirow[t]{5}{*}{$\mathrm{S}_{3}$} & $6.19^{5}(5.31)$ & $\mathrm{HOMO}-2 \rightarrow \mathrm{LUMO}+1$ & 0.20 & 0.08 \\
\hline & & HOMO- $1 \rightarrow$ LUMO & 0.41 & 0.34 \\
\hline & & HOMO-1 $\rightarrow$ LUMO+5 & -0.15 & 0.04 \\
\hline & & $\mathrm{HOMO} \rightarrow \mathrm{LUMO}$ & 0.13 & 0.03 \\
\hline & & $\mathrm{HOMO} \rightarrow \mathrm{LUMO}+1$ & 0.49 & 0.47 \\
\hline (b) & Energy (eV) & Excitation configuration from $\mathrm{D}_{0}$ & Coefficient & Weight \\
\hline $\mathrm{D}_{0}$ & $8.46^{35}$ & Ground configuration & & \\
\hline $\mathrm{D}_{1}$ & 9.12 & HOMO- $1 \rightarrow \mathrm{HOMO}$ & 1.00 & 1.00 \\
\hline \multirow[t]{3}{*}{$\mathrm{D}_{2}$} & 10.76 & HOMO- $1 \rightarrow$ LUMO & 0.18 & 0.03 \\
\hline & & $\mathrm{HOMO} \rightarrow \mathrm{LUMO}$ & 0.27 & 0.07 \\
\hline & & HOMO-2 $\rightarrow \mathrm{HOMO}$ & 0.94 & 0.88 \\
\hline
\end{tabular}

Table 1 Energies and configurations of the lowest electronic states of styrene (a) and the styrene cation (b). The energies are all relative to $S_{0}$. The configurations of the electronically excited states of styrene and the styrene cation were calculated at the TDDFT HCTH/6-311+G* level and are expressed in terms of the orbitals for the

\footnotetext{
* For the excited states of the cation, the total spin of the wavefunction was not constrained; however, the expectation values of $S^{2}$ for the lowest five cation excited state wavefunctions were $0.75-0.78$, corresponding to doublets.
} 
ground state wavefunctions plotted in figure 2. (a) The values for the $\mathrm{S}_{1}{ }^{7}$ and $\mathrm{S}_{2}{ }^{8}$ energies are the experimentally determined band origins. For comparison, the values calculated at the TDDFT HCTH/6-311+G* level are given in brackets. The value for the $\mathrm{S}_{3}$ energy is from a CASPT2 calculation ${ }^{5}$ which is more accurate than our calculated value. Due to the nature of the wavefunction from the TDDFT calculation, the sum of the squares of the configuration coefficients for each excited state is approximately 0.5 for neutral styrene. The values quoted for the weights are the squares of the coefficients, multiplied by a factor of 2 . (b) The value for the $\mathrm{D}_{0}$ energy relative to $S_{0}$ is the experimentally determined first ionisation potential. ${ }^{35}$ The values for the $D_{1}$ and $D_{2}$ energies were obtained by adding our TDDFT HCTH/6-311+G* vertical excitation energies for the cation to this. The sum of the squares of the configuration coefficients for each excited state of the styrene cation is approximately 1.0. The values quoted for the weights are the squares of the coefficients. We have not found any experimental data with which to compare our TDDFT HCTH/6$311+\mathrm{G}^{*}$ calculated energies of $\mathrm{D}_{1}$ and $\mathrm{D}_{2}$; however, Stolow and coworkers calculated values of $10.65 \mathrm{eV}$ and $10.94 \mathrm{eV}$ at the TDDFT B3LYP/6-311+G* level ${ }^{18}$ which are similar.

One-electron ionisation correlations can be determined by inspection of tables 1(a) and (b). The electronic configuration of $\mathrm{D}_{0}$ has a singly occupied highest occupied molecular orbital (HOMO, illustrated in figure 2) while the electronic configuration of $\mathrm{D}_{1}$ has a lower energy singly occupied molecular orbital, labeled HOMO-1, and a pair of electrons in the HOMO. We approximate (based on table 1(b)) that the electronic configuration of $\mathrm{D}_{2}$ corresponds to a single electron in HOMO-2 and a pair of electrons in each of HOMO and HOMO-1.

We assume that ionisation by ultraviolet light involves removing an electron in the highest energy orbital (Koopmans' approximation). From $\mathrm{S}_{1}$ (table 1a), this implies an approximately equal probability for removing an electron from LUMO and LUMO+1: removal from LUMO would leave singly occupied HOMO-1, and correlates with the $\mathrm{D}_{1}$ state for the cation; while removal from LUMO+1 would leave a singly occupied HOMO, corresponding to the cation $\mathrm{D}_{0}$ state. Thus using Koopmans' approximation at fixed Franck Condon geometry, we correlate $S_{1}$ with the $D_{0}$ and $D_{1}$ states of the cation at different excitation energies, and $S_{2}$ almost exclusively with $D_{0}$ using the same method, while $S_{3}$ correlates with $D_{0}, D_{1}$ and $D_{2}$ states of the cation. Approximate ionisation probabilities (not intensities) can be obtained by summing the weights of the neutral configurations that correlate with a particular electronic state of the cation, and these are listed in table 2. 


\begin{tabular}{c|cc}
\hline Neutral state & Cation state & Approximate transition probability \\
\hline \multirow{2}{*}{$\mathrm{S}_{1}$} & $\mathrm{D}_{0}$ & 0.5 \\
& $\mathrm{D}_{1}$ & 0.5 \\
$\mathrm{~S}_{2}$ & $\mathrm{D}_{0}$ & 0.8 \\
& $\mathrm{D}_{1}$ & 0.1 \\
$\mathrm{~S}_{3}$ & $\mathrm{D}_{0}$ & 0.5 \\
& $\mathrm{D}_{1}$ & 0.4 \\
& $\mathrm{D}_{2}$ & 0.1 \\
\hline
\end{tabular}

Table 2 Approximate ionisation correlations between excited singlet states of styrene and the lowest doublet states of the styrene cation.

We assume that vibrational energy is conserved in the transition from the excited state to the cation. This assumption, together with the electronic correlations described above and summarized in table 2, allows approximate photoelectron band energies to be predicted between excited states, and thus the TRPES bands can be assigned to first order, as illustrated in figure 1. Such an approximate assignment method has been reported in the literature as an analysis tool for time-resolved photoionisation studies of styrene and other molecules ${ }^{18,24,29}$ and provides theoretical band energies that agree well with those observed.

\section{Experiment}

The experimental apparatus consists of a femtosecond laser system, molecular beam and velocity map imaging spectrometer. The commercial femtosecond laser system consists of an ultrafast oscillator and amplifier producing a $1 \mathrm{kHz}$ train of $2.5 \mathrm{~mJ}$ pulses of 35 fs duration and a central wavelength of $795 \mathrm{~nm}$. For most experiments, approximately $70 \%$ of the light from the amplifier pumps two optical parametric amplifiers (OPAs) to generate tunable infrared (IR) radiation between $1200 \mathrm{~nm}$ and $2000 \mathrm{~nm}$. The signal from each OPA is then mixed with the residual pump light to produce visible light between $470 \mathrm{~nm}$ and $600 \mathrm{~nm}$ which is subsequently frequency doubled to produce light at $254 \mathrm{~nm}$ and $240 \mathrm{~nm}$. The bandwidth of the UV light is $150-180 \mathrm{~cm}^{-1}$. For some experiments, $35 \%$ of the light from the amplifier pumps one 
OPA and $35 \%$ of the light from the amplifier is frequency-quadrupled to generate light around $199 \mathrm{~nm}$. The pulse energy of the frequency-quadrupled light is attenuated to be $<1 \mu \mathrm{J}$.

For all the experiments described in this paper we employ two pulses that are referred to as pump and probe pulses. The temporal delay between the pump and probe pulses is set by a computer-controlled mechanical translation stage. Both beams are combined at a thin $(1 \mathrm{~mm})$ dichroic mirror and focused collinearly into a pulsed molecular beam of styrene, in the source region of a photoelectron imaging spectrometer, using a $250 \mathrm{~mm}$ fused silica lens. The cross-correlation of the various UV pump and probe pulses lies in the range 175-290 fs, measured by non-resonant ionisation of NO. The pulse energies of the pump and probe are attenuated to $<1 \mu \mathrm{J}$ to ensure that no more than 20 electrons are generated by either the pump or probe alone, minimizing unwanted multiphoton ionisation and space-charge effects.

The photoelectron imaging spectrometer consists of two differentially pumped chambers separated by a $4 \mathrm{~mm}$ home-made skimmer. The source chamber houses the pulsed molecular beam (described below) and is pumped by a $2000 \mathrm{l} / \mathrm{s}$ maglev turbomolecular pump maintaining a base pressure of $8 \times 10^{-8}$ mbar when the pulsed molecular beam is off and less than $2 \times 10^{-7}$ mbar when the pulsed molecular beam is running. The detection chamber contains a velocity map imaging (VMI) spectrometer and a liquid nitrogen cold trap and is pumped by a $1000 \mathrm{l} / \mathrm{s}$ turbomolecular pump maintaining a background pressure of $9 \times 10^{-8}$ mbar, which rises to $1 \times 10^{-7}$ mbar when the molecular beam is running. The VMI spectrometer is based on the Eppink and Parker design ${ }^{36}$ and consists of a set of three polished parallel field plates (repeller, extractor, ground) separated by $15 \mathrm{~mm}$. The extractor voltage was approximately 0.7 of the repeller voltage, which was set to $-1500 \mathrm{~V}$ or $-2500 \mathrm{~V}$ for this work. The photoelectrons are accelerated through a $40 \mathrm{~cm}$ field-free region (surrounded by a layer of $\mu$-metal shielding) and collected by a commercial imaging detector (Photek) comprising a pair of microchannel plates backed by a phosphor coated optical fibre bundle. A CCD camera detects the light transmitted through the fibre bundle and the images are collected on a computer. For each pump-probe delay, we collect pump only, probe only and pump + probe images, in sequence, for 20 seconds each. This is repeated several times (typically 30) for a total of 30 minutes for each time step. This helps reduce the effects of any long-term drift in power due to laser instabilities. The pump only and probe only signals are subtracted from the pump + probe signal to eliminate single colour contributions from the images. Photoelectron spectra are recovered from the raw images using the image inversion program pBasex. ${ }^{37}$ The resolution of the photoelectron imaging detector is defined in terms of the resolution of the photoelectron velocity, $\Delta v$. Since the photoelectron 
kinetic energy $\propto v^{2}$, the energy resolution is better for the lower kinetic energy photoelectrons. In this work, the resolution of the imaging detector as defined by the FWHM of the peaks observed in the (2+1) REMPI ionisation of xenon is $\sim 90 \mathrm{meV}$ at $1.46 \mathrm{eV}$ and $165 \mathrm{meV}$ at $2.76 \mathrm{eV}$.

Experiments were performed using a pulsed molecular beam of styrene created by passing helium at very low pressure $(<100 \mathrm{mBar})$ through liquid styrene (Alfa Aesar 99.5\%, stabilised with 4-tert-butyl-catechol) into an Even-Lavie pulsed valve. The valve was operated at $500 \mathrm{~Hz}$ (to minimize overheating) and held open for 10-13 $\mu$ s. The valve was mounted $135 \mathrm{~mm}$ from the entrance of a skimmer that separated the source and detector chambers, and the distance between the skimmer entrance and the interaction region of the VMI spectrometer was $210 \mathrm{~mm}$.

\section{Results}

We present two experiments investigating the photoinduced dynamics of styrene following excitation to the two lowest excited singlet states. The first experiment tracks the population through $\mathrm{S}_{2} / \mathrm{S}_{1}$ and subsequently $\mathrm{S}_{1} / \mathrm{S}_{0}$ CIs, following excitation to $\mathrm{S}_{2}$ with $\sim 0.3 \mathrm{eV}\left(2300 \mathrm{~cm}^{-1}\right)$ excess vibrational energy. The second experiment tracks the flow of population through an $S_{1} / S_{0} C I$, following direct excitation to $S_{1}$ with $\sim 0.6 \mathrm{eV}\left(4600 \mathrm{~cm}^{-1}\right)$ excess vibrational energy. The dynamics are monitored using TRPES.

In figure 3, we present photoelectron spectra recorded following excitation of styrene with femtosecond laser light at $240 \mathrm{~nm}$, corresponding to $\sim 0.3 \mathrm{eV}$ excess vibrational energy in $\mathrm{S}_{2}$, which after passing through an $\mathrm{S}_{2} / \mathrm{S}_{1}$ CI corresponds to $\sim 0.9 \mathrm{eV}$ excess vibrational energy in $S_{1}$ (see table 1). The probe wavelength is centered at $254 \mathrm{~nm}$, providing a combined energy of $10.0 \mathrm{eV}$, i.e. above $\mathrm{D}_{0}$ and $\mathrm{D}_{1}$ (see table 1(b)). Figure 3(a) is the pump-probe spectrum recorded at $t=0$ and shows that there are three overlapping bands. Using the ionisation correlations presented in table 2, and assuming that any excess vibrational energy is conserved, to a first approximation, during the ionisation process, bands around $1.2 \mathrm{eV}, 0.5 \mathrm{eV}$ and $0.1 \mathrm{eV}$ are assigned to the $S_{2}-D_{0}, S_{1}-D_{0}$ and $S_{1}-D_{1}$ ionisation processes, respectively.

The dynamics occur over a wide range of timescales, so we split our presentation of the time-resolved photoelectron spectra into two parts: time-resolved photoelectron spectra from $0-60 \mathrm{ps}$ are presented in figure 3(b) and time-resolved photoelectron spectra from -100-250 fs are presented in figure 3(c). The $S_{2}-D_{0}$ band $(1.2 \mathrm{eV})$ has a maximum around $t=0$ and decays within a few hundred femtoseconds. In contrast, the $S_{1}-D_{0}$ and $S_{1}-D_{1}$ bands $(0.5 \mathrm{eV}$ and $0.1 \mathrm{eV})$ only reach their maxima once the $S_{2^{-}}$ 
$\mathrm{D}_{0}$ band has decayed, and are then relatively long-lived, decaying on the timescale of tens of picoseconds. The integrated intensity of each band can be considered as an approximate measure of the population being projected from the excited state onto the ionisation continuum. Thus, a more quantitative picture of the dynamics can be obtained by plotting the time-dependence of the integrated intensity of each band individually. These integrated intensities are presented in figure 4. Again, we present the data in two parts: 0-50 ps integrated band intensities are presented in figure 4(a) and 0-500 fs integrated band intensities are presented in figure 4(b).

To deconvolute the dynamics of the molecule from the resolution of the instrument (sometimes called the instrument response function) we have performed a global fit to the temporal dynamics of each band. The fitted function is a convolution of the Gaussian laser pulse with an exponential decay and is plotted as a solid line in figures 4(a) and (b). The cross-correlation FWHM, which was determined from an independent measurement of the cross-correlation intensity by non-resonant ionisation of NO, was $203 \pm 25 \mathrm{fs}$, but because of the uncertainty it was also included in the fit. The expression used to fit the data presented in figures 4(a) and (b) is:

$$
f(t)=N\left(1+\operatorname{erf}\left(\left(t-t_{0}-\left(\sigma^{2} / \tau\right)\right) /(\sigma \sqrt{2})\right)\right) \exp \left(-t / \tau+t_{0} / \tau+\sigma^{2} /\left(2 \tau^{2}\right)\right),
$$

where $t$ is the pump-probe delay. The parameters were obtained by fitting all three decay curves together: time zero $\left(t_{0}\right) ; 1 / e$ lifetime $(\tau)$; the variance of the crosscorrelation pulse width $\left(\sigma^{2}\right)$. The full-width at half-maximum for the crosscorrelation is related to the standard deviation, $F W H M=\sigma 2 \sqrt{2 \ln 2}$, and is determined to be 235 fs from the fit. See supplementary information for the derivation of equation (1). The fitted value for $t_{0}$ lies within the estimated experimental error from our measured cross-correlation of the pump and probe pulses of \pm 25 fs. All the fit parameters are presented in Table 3.

\begin{tabular}{c|cccc}
\hline & $\Delta E(\mathrm{eV})$ & $\tau(\mathrm{fs})$ & $\sigma(\mathrm{fs})$ & $t_{0}(\mathrm{fs})$ \\
\hline $\mathrm{S}_{2}-\mathrm{D}_{0}$ & $0.9-1.4$ & 13 & 100 & -23 \\
$\mathrm{~S}_{1}-\mathrm{D}_{0}$ and $\mathrm{S}_{1}-\mathrm{D}_{1}$ & $0-0.15,0.15-0.8$ & 18820 & 100 & -23 \\
\hline
\end{tabular}

Table 3 The fit parameters for equation (1) obtained for the data presented in figures 4(a) and (b). 
From the fit, the loss of population from $S_{2}$ occurs exponentially with a $1 / e$ timescale of $13 \mathrm{fs}$ and the concomitant gain in $\mathrm{S}_{1}$ population occurs with the same $1 / e$ timescale of $13 \mathrm{fs}$. The subsequent loss of population from $S_{1}$ occurs exponentially with a 1/e timescale of 19 ps. The fact that the loss of population from $S_{2}$ and the gain of population on $S_{1}$ occur on the same timescale is evidence that we are observing ultrafast (essentially instantaneous on the timescale of our experiment) decay through the $S_{2} / S_{1}$ CI. The upper limit for the $1 / e$ decay of $S_{2}$ and rise of $S_{1}$ is approximately 45 fs (obtained from the fit). Despite the huge amount of excess vibrational energy in $S_{1}$ $(\sim 0.9 \mathrm{eV})$, the subsequent loss of population from $S_{1}$ is three orders of magnitude slower than decay through an $\mathrm{S}_{2} / \mathrm{S}_{1} \mathrm{CI}$, suggesting that there is effectively a barrier on the $S_{1}$ potential energy surface preventing ultrafast IC through an $S_{1} / S_{0} C I$.

To investigate this further, we performed a new set of experiments in which we excited just below the onset of absorption to the $S_{2}$ state, populating $S_{1}$ with the maximum possible excess vibrational energy by direct excitation from $S_{0}(\sim 0.6 \mathrm{eV})$. Initially, the probe wavelength was selected to be $240 \mathrm{~nm}$ so that we accessed the same region of the ionisation continuum as in the first set of experiments; however, the much larger cross-section for excitation to $S_{2}$ at $240 \mathrm{~nm}$ resulted in the photoelectron spectrum being dominated by the $240 \mathrm{~nm}$ pump $+254 \mathrm{~nm}$ probe process at short times, i.e. within the cross-correlation. This problem was solved by selecting a much shorter probe wavelength so that the unwanted bands resulting from $\mathrm{S}_{2}$ excitation were well-separated from the bands arising from $\mathrm{S}_{1}$ excitation.

In figure 5, we present photoelectron spectra recorded following excitation of styrene with femtosecond laser light at $254 \mathrm{~nm}$, corresponding to $\sim 0.6 \mathrm{eV}$ excess vibrational energy in $S_{1}$. The probe wavelength is centered at $199 \mathrm{~nm}$, providing a combined energy of $11.1 \mathrm{eV}$, i.e. above $\mathrm{D}_{0}, \mathrm{D}_{1}$ and $\mathrm{D}_{2}$ (see table $1(\mathrm{~b})$ ). Figure 5(a) is the pumpprobe spectrum recorded at $t=0$ and there are three overlapping bands. As before, using the ionisation correlations presented in table 2, bands around $1.75 \mathrm{eV}$ and 1.0 $\mathrm{eV}$ are assigned to the $\mathrm{S}_{1}-\mathrm{D}_{0}$ and $\mathrm{S}_{1}-\mathrm{D}_{1}$ ionisation processes, respectively. There is another band around $0.4 \mathrm{eV}$ that is only present in the photoelectron spectrum during the time that the pump and probe pulses overlap temporally. This band is attributed to the unwanted $S_{3}-D_{0}$ ionisation process and is a result of excitation to $S_{3}$ by the $199 \mathrm{~nm}$ probe pulse. Fortunately, the $S_{3}-D_{0}$ band is reasonably well-separated, energetically, from the $S_{1}-D_{0}$ and $S_{1}-D_{1}$ bands.

The integrated intensities of the $S_{1}-D_{0}$ and $S_{1}-D_{1}$ bands are plotted as before as a function of pump-probe delay in figure 6 . The decay rates of both the $S_{1}-D_{0}$ and $S_{1}-D_{1}$ bands are approximately the same at long times (i.e. beyond the cross-correlation time of the two laser pulses) and are also the same as the decay rate of the total integrated 
electron count. The higher energy $S_{1}-D_{0}$ band at $1.75 \mathrm{eV}$ is well-separated from the $\mathrm{S}_{3}-\mathrm{D}_{0}$ band at $0.4 \mathrm{eV}$. We can therefore fit this band to the same convolution of the instrument response function and an exponential decay described above. The experimentally determined cross-correlation was $287 \pm 40 \mathrm{fs}$, with an error in $t_{0}$ of \pm 100 fs. The $S_{1}-D_{1}$ band has some overlap with the unwanted $S_{3}-D_{0}$ band at short times when the pump and probe pulses overlap temporally. This results in an artificial peak in the integrated $S_{1}-D_{1}$ band at short times. The effect of the $S_{3}$ component can be incorporated in the fit by the addition of another Gaussian that matches the laser cross-correlation width.

The $S_{1}-D_{0}$ data presented in figure 6 is fit using equation (1), while the $S_{1}-D_{1}$ data in figure 6 is fit using

$$
\begin{aligned}
& f(t)=N\left(1+\operatorname{erf}\left(\left(t-t_{0}-\left(\sigma^{2} / \tau\right)\right) /(\sigma \sqrt{2})\right)\right) \exp \left(-t / \tau+t_{0} / \tau+\sigma^{2} /\left(2 \tau^{2}\right)\right) \\
& +N_{2} \exp \left(\left(-t-t_{0}\right)^{2} /\left(\sigma^{2} \sqrt{2}\right)\right)
\end{aligned}
$$

where all symbols have the same meaning as in equation (1). See supplementary information for a derivation of equation (2). The parameters were obtained by independently fitting the two decay curves. The parameters obtained from independent fits to both the $S_{1}-D_{0}$ and $S_{1}-D_{1}$ bands are presented in Table 4 .

\begin{tabular}{l|ccccc}
\hline & $\Delta E(\mathrm{eV})$ & $\tau(\mathrm{fs})$ & $\sigma(\mathrm{fs})$ & $t_{0}(\mathrm{fs})$ & $N / N_{2}$ \\
\hline $\mathrm{S}_{1}-\mathrm{D}_{0}$ & $1.5-2.2$ & 3960 & 115 & -71 & \\
$\mathrm{~S}_{1}-\mathrm{D}_{1}$ & $0.8-1.5$ & 3220 & 115 & -71 & $0.5 / 0.3$ \\
\hline
\end{tabular}

Table 4 The fit parameters obtained for equations (1) and (2) for the data presented in figure 6 . The value for $t_{0}$ lies within our estimated experimental error of the absolute time-zero as measured via the cross-correlation of the pump and probe pulses of \pm 100 fs.

From the fit, it is apparanet that the loss of population from $S_{1}$ occurs exponentially with a $1 / e$ timescale between $3.2 \mathrm{ps}$ and $4 \mathrm{ps}$. Despite the fact that styrene has around $\sim 0.6 \mathrm{eV}$ excess vibrational energy in $\mathrm{S}_{1}$ when excited directly from $\mathrm{S}_{0}$ with light at $254 \mathrm{~nm}$, compared to $\sim 0.9 \mathrm{eV}$ excess vibrational energy when populated indirectly via 
the $S_{2} / S_{1}$ conical intersection following $S_{2}$ excitation with light at $240 \mathrm{~nm}$, the decay from $S_{1}$ is of the order of five times faster.

\section{Discussion}

Experimentally, we find that $S_{1} / S_{0}$ radiationless decay in styrene is slower when the $\mathrm{S}_{1}$ state is accessed via $\mathrm{S}_{2}$ compared to direct access on $\mathrm{S}_{1}$, even when there is sufficient excess vibrational energy in both cases. Based on this result, we suggest the following outline mechanism.

- $\mathrm{S}_{1}$ radiationless decay is barrier activated (picosecond timescale), via a conical intersection that does not occur along the initial geometry relaxation coordinate on this state.

- $\mathrm{S}_{2}$ radiationless decay is barrierless (femtosecond timescale): the initial geometry relaxation leads directly to a conical intersection with $S_{1}$.

- Geometry changes as a result of ultrafast decay from $S_{2}$ result in $S_{1}$ population that is 'further' from an $S_{1} / S_{0}$ crossing, which must be accessed indirectly along a different coordinate, leading to a difference in $\mathrm{S}_{1}$ decay times.

We invoke conical intersections in our discussion rather than the more traditional Fermi's Golden Rule type IC due to the time scales involved. The rate of Fermi's Golden Rule type IC generally decreases with increasing vibrational energy, ${ }^{38}$ and previous lifetime measurements of the $S_{1}$ state with low excess energy have shown lifetimes of the order of several tens of nanoseconds. ${ }^{15,39-40}$ Therefore, it is extremely unlikely that the rate of $S_{1} / S_{0}$ IC would increase by three orders of magnitude with increasing excess energy, as we observe, without the appearance of a new IC mechanism.

The observations can be rationalised in terms of a model based on the excited state structures located in reference 15. In this CASSCF/4-31G study, CIs between $S_{1} / S_{0}$ were found at $5.41 \mathrm{eV}$ and $6.03 \mathrm{eV}$ above the $\mathrm{S}_{0}$ minimum (these energies are certainly over-estimated, and cannot be compared directly with our highest experimental excitation energy which was $5.2 \mathrm{eV}(240 \mathrm{~nm}))$. The geometry of the higher-energy $S_{1} / S_{0} C I$ was determined to be similar to that of a twisted $S_{2} / S_{1}$ CI (where $S_{2}$ was a covalent excited state ${ }^{41}$ ), shown as the upper structure in figure 7 : the only difference is that for this twisted $S_{1} / S_{0} C I$, the ethylene carbon closest to the benzene ring is raised markedly out of plane. In contrast, the lower energy $S_{1} / S_{0} C I$ appears similar to the prefulvene geometry of the $\mathrm{S}_{1} / \mathrm{S}_{0} \mathrm{CI}$ in benzene, i.e. the ethylene 
and ethyl-benzene $\mathrm{CC}$ bond lengths are similar to those of the $\mathrm{S}_{0}$ minimum, but the benzene ring has undergone a prefulvene-like out-of-plane distortion, shown as the lower structure in figure 7 .

If the two CIs $\left(\mathrm{S}_{2} / \mathrm{S}_{1}\right.$ and $\left.\mathrm{S}_{1} / \mathrm{S}_{0}\right)$ involved in the decay dynamics observed in our 240 $\mathrm{nm} \mathrm{S}_{2}$ pump experiments were similar geometrically, and located along the same reaction path starting at the Franck Condon geometry, one would expect that decay from $S_{2}$ to $S_{1}$ and subsequent decay from $S_{1}$ to $S_{0}$ would both be fast; indeed, faster than decay directly from $S_{1}$ alone. Because this is not the case in our $240 \mathrm{~nm}$ pump experiment, we deduce that in our experiments we observe decay via an $\mathrm{S}_{1} / \mathrm{S}_{0} \mathrm{CI}$ having a very different geometry to that at which $S_{1}$ is accessed. Based on the outline mechanism above, one interpretation is that the initial decay from $S_{2}$ to $S_{1}$ is through a twisted CI and the subsequent decay from $S_{1}$ is through a very different prefulvenelike CI, as shown in figure 7. At present, we cannot say whether both decay sequences we have studied - from $S_{2}$ and separately from direct $S_{1}$ excitation - result in different rates for accessing a common region of an $S_{1} / S_{0}$ intersection seam such as the prefulvene one, or whether different crossings are involved in each case. This is of course only one interpretation based on the specific structures described in reference 15. Nonetheless, we expect any detailed interpretation to be consistent with this general model. To check our provisional interpretation would require further testing through accurate calculations of the excited state potential energy surfaces, including all relevant geometry changes and crossings.

\section{Summary}

TRPES has been used to study the ultrafast decay dynamics of styrene following excitation into the two lowest excited singlet states. Internal conversion between $S_{2}$ and $S_{1}$ and between $S_{1}$ and $S_{0}$, have both been observed. The dynamics observed in $S_{1}$ are quite different depending on whether the state is populated directly by the absorption of a photon, or indirectly following IC from $\mathrm{S}_{2}$. Contrary to what might be expected, the more energetic molecule produced via IC from $S_{2}$ has a much longer $S_{1}$ lifetime than its less energetic counterpart. The observations have been explained in terms of geometries encountered by the molecule as it relaxes electronically back to the ground state, and the large geometry changes that would need to occur to allow IC to $S_{1}$ and subsequently to $S_{0}$. Hopefully, these results will stimulate interest in using quantum molecular dynamics calculations to determine the decay path from the $S_{2} / S_{1}$ CI through the $S_{1} / S_{0} C I$ to $S_{0}$, based on accurate potential energy surfaces for all of the excited states involved. 


\section{Acknowledgements}

We acknowledge support from the EPSRC, The Ramsay Trust (R.S.M.) the European Marie Curie Initial Training Network Grant No. CA-ITN-214962-FASTQUAST (R.S.) and UCL. 


\section{Figure Captions}

\section{Figure 1}

Schematic energy level diagram showing the four lowest, singlet states of styrene $\left(\mathrm{S}_{0}\right.$, $\left.S_{1}, S_{2}, S_{3}\right)$ and the three lowest, doublet states $\left(D_{0, d 1, d 2}\right)$ of the styrene cation. In the experiments described in this paper the pump pulse excites styrene with $\sim 0.3 \mathrm{eV}$ excess vibrational energy in $S_{2}$ (shown) or $\sim 0.6 \mathrm{eV}$ excess vibrational energy in $S_{1}$ (not shown). The timescale for $\mathrm{S}_{2} / \mathrm{S}_{1}$ IC and $\mathrm{S}_{1} / \mathrm{S}_{0}$ IC are marked $\Delta \mathrm{t}_{1}$ and $\Delta \mathrm{t}_{2}$, respectively. There is an approximate propensity for the vibrational quantum number to be conserved during photoionisation; therefore, since $E_{\text {vib }}\left(\mathrm{S}_{1}\right)<E_{\text {vib }}\left(\mathrm{S}_{2}\right)$ we would expect the thresholds for ionisation to be in the same order, $I_{\text {threshold }}\left(\mathrm{S}_{1}\right)<I_{\text {threshold }}\left(\mathrm{S}_{2}\right)$ for a given doublet state of the cation. For a given probe energy, different electronic excited states of styrene will be projected onto different electronic states of the cation which will map onto different (but overlapping) portions of the photoionisation continuum (cartoon photoelectron spectrum on right hand side of figure).

\section{Figure 2}

Orbitals for the ground state wavefunctions of neutral styrene and the styrene cation used as reference orbitals for the expansion of the excited state wavefunctions calculated at the DFT HCTH/6-311+G* level. The horizontal dashed line separates occupied orbitals from unoccupied orbitals in the electronic ground states of the neutral and cation species.

\section{Figure 3}

Photoelectron spectra recorded following excitation of styrene with femtosecond laser light at $240 \mathrm{~nm}$, corresponding to $\sim 0.3 \mathrm{eV}$ excess vibrational energy in $\mathrm{S}_{2}$, and a probe wavelength centered at $254 \mathrm{~nm}$. The $t=0$ photoelectron spectrum is presented in (a), highlighting bands around $1.2 \mathrm{eV}\left(\mathrm{S}_{2}-\mathrm{D}_{0}\right), 0.5 \mathrm{eV}\left(\mathrm{S}_{1}-\mathrm{D}_{0}\right)$ and $0.1 \mathrm{eV}\left(\mathrm{S}_{1}-\mathrm{D}_{1}\right)$. Timeresolved photoelectron spectra from 0-60 ps are presented in (b) and time-resolved photoelectron spectra from $-100-250$ fs are presented in (c).

\section{Figure 4}

Partial integrated photoelectron yields following excitation of styrene with femtosecond laser light at $240 \mathrm{~nm}$, corresponding to $\sim 0.3 \mathrm{eV}$ excess vibrational energy in $S_{2}$, for 0-50 ps (a) and 0-500 fs (b). Open triangles correspond to $S_{1}-D_{1}$ ionisation, open circles correspond to $S_{1}-D_{0}$ ionisation and filled circles correspond to $\mathrm{S}_{2}-\mathrm{D}_{0}$ ionisation. The solid lines are global fits to the experimental data (see text and table 3).

\section{Figure 5}


Photoelectron spectra recorded following excitation of styrene with femtosecond laser light at $254 \mathrm{~nm}$, corresponding to $\sim 0.6 \mathrm{eV}$ excess vibrational energy in $\mathrm{S}_{1}$, and a probe wavelength centered at $199 \mathrm{~nm}$. The $t=0$ photoelectron spectrum is presented in (a), highlighting bands around $1.75 \mathrm{eV}\left(\mathrm{S}_{1}-\mathrm{D}_{0}\right), 1.0 \mathrm{eV}\left(\mathrm{S}_{1}-\mathrm{D}_{1}\right)$ and $0.4 \mathrm{eV}\left(\mathrm{S}_{3}-\mathrm{D}_{0}\right)$. Timeresolved photoelectron spectra from $-100-1200$ fs are presented in (b).

\section{Figure 6}

Partial integrated photoelectron yields following excitation of styrene with femtosecond laser light at $254 \mathrm{~nm}$, corresponding to $\sim 0.6 \mathrm{eV}$ excess vibrational energy in $S_{1}$, for 0-6 ps (a) and 0-1400 fs (b). Open triangles correspond to $S_{1}-D_{1}$ ionisation, open circles correspond to $S_{1}-D_{0}$ ionisation. The filled circles in (a) correspond to the total integrated photoelectron yield. The data points and error bars are the average and \pm 2 standard deviations of electron counts collected for 15000 laser shots. The solid lines are global fits to the experimental data (see text and table 4).

\section{Figure 7}

Cartoon illustrating geometries of the $S_{1} / S_{0}$ and $S_{2} / S_{1}$ CIs and the non-radiative decay routes following excitation of styrene with femtosecond laser light at $240 \mathrm{~nm}$, corresponding to $\sim 0.3 \mathrm{eV}$ excess vibrational energy in $\mathrm{S}_{2}$ and $\sim 0.9 \mathrm{eV}$ excess vibrational energy in $\mathrm{S}_{1}$ after passing through $\mathrm{S}_{2} / \mathrm{S}_{1} \mathrm{CI}$. 


\section{References}

[1] A. Migani and M. Olivucci in Conical Intersections and Organic Reaction Mechanisms, Vol. (Ed. D. R. Y. a. H. K. Wolfgang Domcke), World Scientific, Singapore, 2004, pp. 271-320.

[2] J. G. Philis, A. Ioannidou and A. A. Christodoulides, Journal of Molecular Spectroscopy 1995, 174, 51-58.

[3] P. Swiderek, M. J. Fraser, M. Michaud and L. Sanche, Journal of Chemical Physics 1994, 100, 70-77.

[4] L. D. Ziegler and C. Carotsis, Chemical Physics Letters 1986, 123, 175-181.

[5] V. Molina, B. R. Smith and M. Merchan, Chemical Physics Letters 1999, 309, 486-494.

[6] J. Wan and H. Nakatsuji, Chemical Physics 2004, 302, 125-134.

[7] J. M. Hollas and T. Ridley, Chemical Physics Letters 1980, 75, 94-98.

[8] D. G. Leopold, R. J. Hemley, V. Vaida and J. L. Roebber, Journal of Chemical Physics 1981, 75, 4758-4769.

[9] J. G. Philis, E. Drougas and A. M. Kosmas, Chemical Physics 2004, 306, 253-263.

[10] T. R. Gilson, J. M. Hollas, E. Khalilipour and J. V. Warrington, Journal of Molecular Spectroscopy 1978, 73, 234-239.

[11] Y. Haas, S. Kendler, E. Zingher, H. Zuckermann and S. Zilberg, Journal of Chemical Physics 1995, 103, 37-47.

[12] J. M. Hollas, E. Khalilipour and S. N. Thakur, Journal of Molecular Spectroscopy 1978, 73, 240-265.

[13] M. J. Bearpark, M. Olivucci, S. Wilsey, F. Bernardi and M. A. Robb, Journal of the American Chemical Society 1995, 117, 6944-6953.

[14] R. Bonneau, Journal of Photochemistry 1979, 10, 439-449.

[15] R. Bonneau, Journal of the American Chemical Society 1982, 104, 2921-2923.

[16] Y. Amatatsu, Journal of Computational Chemistry 2002, 23, 950-956.

[17] V. Molina, M. Merchan, B. O. Roos and P. A. Malmqvist, Physical Chemistry

Chemical Physics 2000, 2, 2211-2217.

[18] S. H. Lee, K. C. Tang, I. C. Chen, M. Schmitt, J. P. Shaffer, T. Schultz, J. G.

Underwood, M. Z. Zgierski and A. Stolow, Journal of Physical Chemistry A 2002, 106, 8979-8991.

[19] A. Stolow, A. E. Bragg and D. M. Neumark, Chemical Reviews 2004, 104, 17191757.

[20] A. Stolow and J. G. Underwood in TIME-RESOLVED PHOTOELECTRON SPECTROSCOPY OF NONADIABATIC DYNAMICS IN POLYATOMIC MOLECULES, Vol. 139 2008, pp. 497-583.

[21] T. Suzuki, Annual Review of Physical Chemistry 2006, 57, 555-592.

[22] T. Suzuki and B. J. Whitaker, International Reviews in Physical Chemistry 2001, 20,313-356.

[23] A. Assion, T. Baumert, J. Helbing, V. Seyfried and G. Gerber, Chemical Physics Letters 1996, 259, 488-494.

[24] V. Blanchet, M. Z. Zgierski, T. Seideman and A. Stolow, Nature 1999, 401, 5254.

[25] C. Z. Bisgaard, O. J. Clarkin, G. R. Wu, A. M. D. Lee, O. Gessner, C. C. Hayden and A. Stolow, Science 2009, 323, 1464-1468.

[26] O. Gessner, E. T. H. Chrysostom, A. M. D. Lee, D. M. Wardlaw, M. L. Ho, S. J. Lee, B. M. Cheng, M. Z. Zgierski, I. C. Chen, J. P. Shaffer, C. C. Hayden and A. Stolow, Faraday Discussions 2004, 127, 193-212. 
[27] O. Gessner, A. M. D. Lee, J. P. Shaffer, H. Reisler, S. V. Levchenko, A. I. Krylov, J. G. Underwood, H. Shi, A. L. L. East, D. M. Wardlaw, E. T. Chrysostom, C. C. Hayden and A. Stolow, Science 2006, 311, 219-222.

[28] C. J. Hammond, K. L. Reid and K. L. Ronayne, Journal of Chemical Physics 2006, 124.

[29] B. Kim, C. P. Schick and P. M. Weber, Journal of Chemical Physics 1995, 103, 6903-6913.

[30] R. S. Minns, D. S. N. Parker, T. J. Penfold, G. A. Worth and H. H. Fielding, PCCP 2010, DOI: 10.1039/c001671c.

[31] D. S. N. Parker, R. S. Minns, T. J. Penfold, G. A. Worth and H. H. Fielding, Chemical Physics Letters 2009, 469, 43-47.

[32] A. D. Boese and N. C. Handy, Journal of Chemical Physics 2001, 114, 54975503.

[33] F. A. Hamprecht, A. J. Cohen, D. J. Tozer and N. C. Handy, Journal of Chemical Physics 1998, 109, 6264-6271.

[34] N. C. Handy and D. J. Tozer, Journal of Computational Chemistry 1999, 20, 106-113.

[35] J. M. Dyke, H. Ozeki, M. Takahashi, M. C. R. Cockett and K. Kimura, Journal of Chemical Physics 1992, 97, 8926-8933.

[36] A. T. J. B. Eppink and D. H. Parker, Review of Scientific Instruments 1997, 68, 3477-3484.

[37] G. A. Garcia, L. Nahon and I. Powis, Review of Scientific Instruments 2004, 75, 4989-4996.

[38] J. Michel and V. Bonacic-Koutecky, Electronic aspects of organic photochemistry, Wiley Interscience, 1990, p.

[39] K. P. Ghiggino, D. Phillips, K. Salisbury and M. D. Swords, Journal of Photochemistry 1977, 7, 141-146.

[40] J. M. Hollas and T. Ridley, Journal of Molecular Spectroscopy 1981, 89, 232253.

[41] M. de Groot and W. J. Buma, Chemical Physics Letters 2007, 435, 224-229. 


\section{Supplementary information}

\section{S1. Time evolution of the excited state population}

We assume Gaussian temporal profiles for the pump and probe pulses:

$$
I(t)=\frac{A}{\sqrt{2 \pi} \sigma} \exp \left(-\frac{1}{2} \frac{\left(t-t_{0}\right)^{2}}{\sigma^{2}}\right)
$$

Neglecting any coherent effects, the population, $f(t)$, in any excited state, $\mathrm{S}_{i}$, varies with time according to the formula :

$$
\frac{d f(t)}{d t}=\Sigma_{0 i} I(t)-k f(t),
$$

where $\Sigma_{0 i}$ is the excitation cross-section from the ground state to the electronically excited state $S_{i}$, and $k$ is the total decay rate of the $S_{i}$ state. This first order differential equation has a known solution:

$$
f(t)=\exp (-k t) \int_{-\infty}^{t} \exp (k x) \Sigma_{0 i} I(x) d x=\exp (-k t) \int_{-\infty}^{t} \exp (k x) \Sigma_{0 i} \frac{A}{\sqrt{2 \pi} \sigma} \exp \left(-\frac{1}{2} \frac{\left(x-t_{0}\right)^{2}}{\sigma^{2}}\right) d x
$$

The convolution of the exponential and the Gaussian functions can be simplified and expressed in terms of the error function (see section S2), thus the time-evolution of the population in the excited state can be described by :

$$
f(t)=\exp (-k t) \Sigma_{0 i} \frac{A}{2} \exp \left(\frac{\sigma^{2} k^{2}}{2}+k t_{0}\right)\left(1+e r f\left(\frac{t-t_{0}-\sigma^{2} k}{\sqrt{2} \sigma}\right)\right)
$$

The decay rate, $k$, is related to the decay time, $\tau$, by $k=1 / \tau$. By substituting for $\tau$ and setting $N=\Sigma_{0 i} A / 2$, we obtain equation (1) in the main paper:

$$
f(t)=N \exp \left(\frac{\sigma^{2}}{2 \tau^{2}}+\frac{t_{0}}{\tau}-\frac{t}{\tau}\right)\left(1+\operatorname{erf}\left(\frac{t-t_{0}-\sigma^{2}}{\tau \sigma \sqrt{2}}\right)\right)
$$

\section{S2. Convolution of the exponential and Gauss functions}

In order to solve the convolution integral,

$$
\int_{-\infty}^{t} \exp (k x) \exp \left(-\frac{1}{2} \frac{\left(x-t_{0}\right)^{2}}{\sigma^{2}}\right) d x=\int_{-\infty}^{t} \exp \left(k x-\frac{1}{2} \frac{\left(x-t_{0}\right)^{2}}{\sigma^{2}}\right) d x
$$

the exponent in equation (5) has to be rearranged as shown below.

$$
\begin{aligned}
& k x-\frac{1}{2} \frac{\left(x-t_{0}\right)^{2}}{\sigma^{2}}=-\frac{1}{2} \frac{\left(x-t_{0}\right)^{2}}{\sigma^{2}}+k\left(x-t_{0}\right)-k t_{0}=-\frac{\left(x-t_{0}\right)^{2}-2 \sigma^{2} k\left(x-t_{0}\right)}{2 \sigma^{2}}+k t_{0}= \\
& =-\frac{\left(x-t_{0}\right)^{2}-2 \sigma^{2} k\left(x-t_{0}\right)+\sigma^{4} k^{2}-\sigma^{4} k^{2}}{2 \sigma^{2}}+k t_{0}=-\frac{\left(x-t_{0}-\sigma^{2} k\right)^{2}}{2 \sigma^{2}}+\frac{\sigma^{2} k^{2}}{2}+k t_{0}
\end{aligned}
$$


To simplify this expression, a new variable, $y$, and an upper integration limit, $t^{\prime}$, is introduced as defined below:

$$
y=\frac{x-t_{0}-\sigma^{2} k}{\sqrt{2} \sigma}, d x=\sqrt{2} \sigma d y, t^{\prime}=\frac{t-t_{0}-\sigma^{2} k}{\sqrt{2} \sigma}
$$

Combining the above mathematical transformations gives the resultant integral:

$$
\int_{-\infty}^{t} \exp \left(k x-\frac{1}{2} \frac{\left(x-t_{0}\right)^{2}}{\sigma^{2}}\right) d x=\exp \left(\frac{\sigma^{2} k^{2}}{2}+k t_{0}\right) \sqrt{2} \sigma \int_{-\infty}^{t^{\prime}} \exp \left(-y^{2}\right) d y
$$

Using the standard integral $\int_{-\infty}^{0} \exp \left(-y^{2}\right) d y=\frac{\sqrt{\pi}}{2}$, expression (7) can now be derived via the error function, $\int_{0}^{t} \exp \left(-y^{2}\right) d y=\frac{\sqrt{\pi}}{2} \operatorname{erf}(t)$, as shown below :

$$
\begin{aligned}
\int_{-\infty}^{t} \exp (k x) \exp \left(-\frac{1}{2} \frac{\left(x-t_{0}\right)^{2}}{\sigma^{2}}\right) d & =\exp \left(\frac{\sigma^{2} k^{2}}{2}+k t_{0}\right) \sqrt{2} \sigma \int_{-\infty}^{t^{\prime}} \exp \left(-y^{2}\right) d y \\
& =\exp \left(\frac{\sigma^{2} k^{2}}{2}+k t_{0}\right) \sqrt{2} \sigma\left(\int_{-\infty}^{0} \exp \left(-y^{2}\right) d y+\int_{0}^{t^{\prime}} \exp \left(-y^{2}\right) d y\right) \\
& =\exp \left(\frac{\sigma^{2} k^{2}}{2}+k t_{0}\right) \sigma\left(\sqrt{\frac{\pi}{2}}+\sqrt{\frac{\pi}{2}} \operatorname{erf}\left(t^{\prime}\right)\right) \\
& =\exp \left(\frac{\sigma^{2} k^{2}}{2}+k t_{0}\right) \sigma \sqrt{\frac{\pi}{2}}\left(1+\operatorname{erf}\left(\frac{t-t_{0}-\sigma^{2} k}{\sqrt{2} \sigma}\right)\right)
\end{aligned}
$$




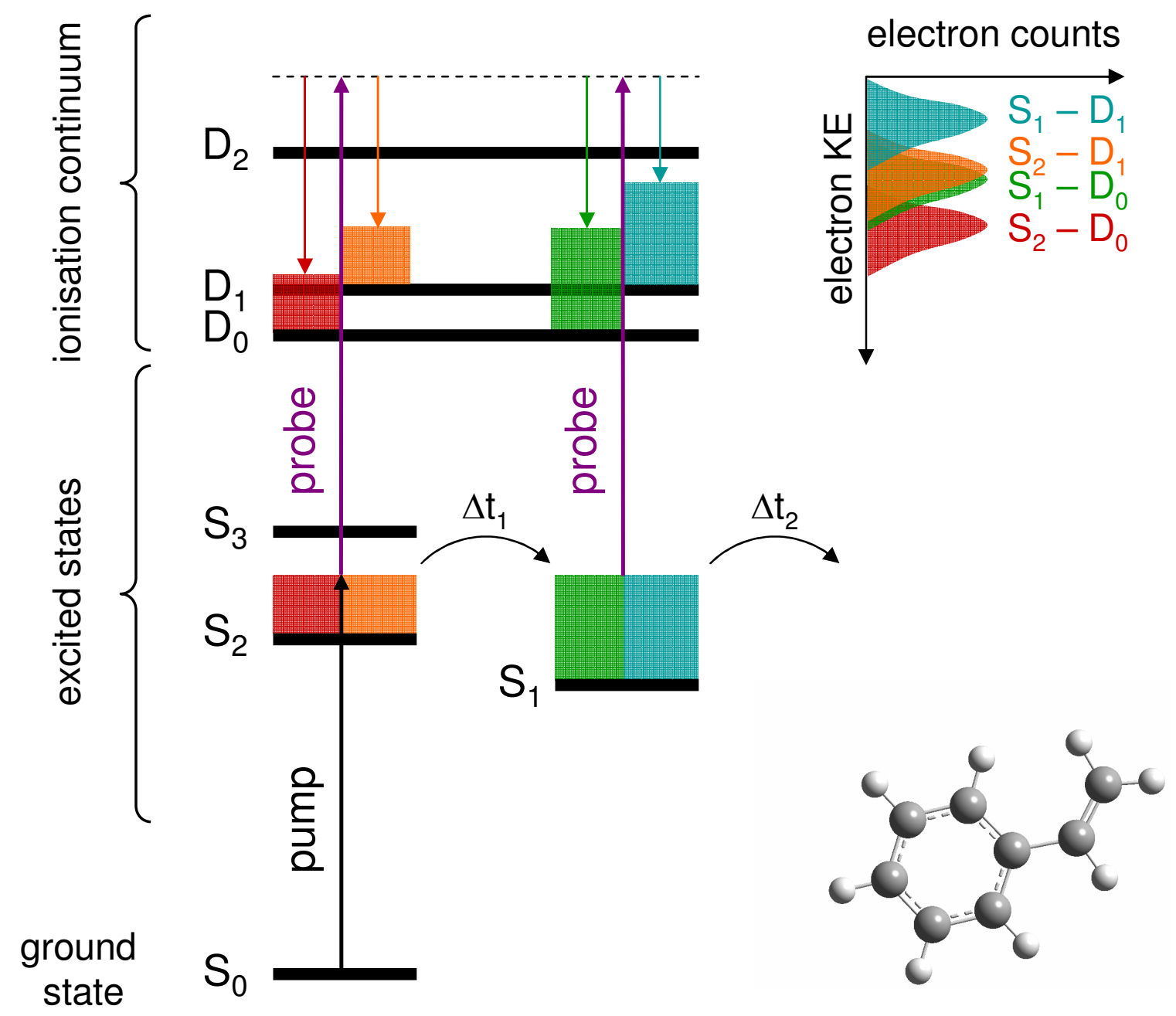

Figure 1 

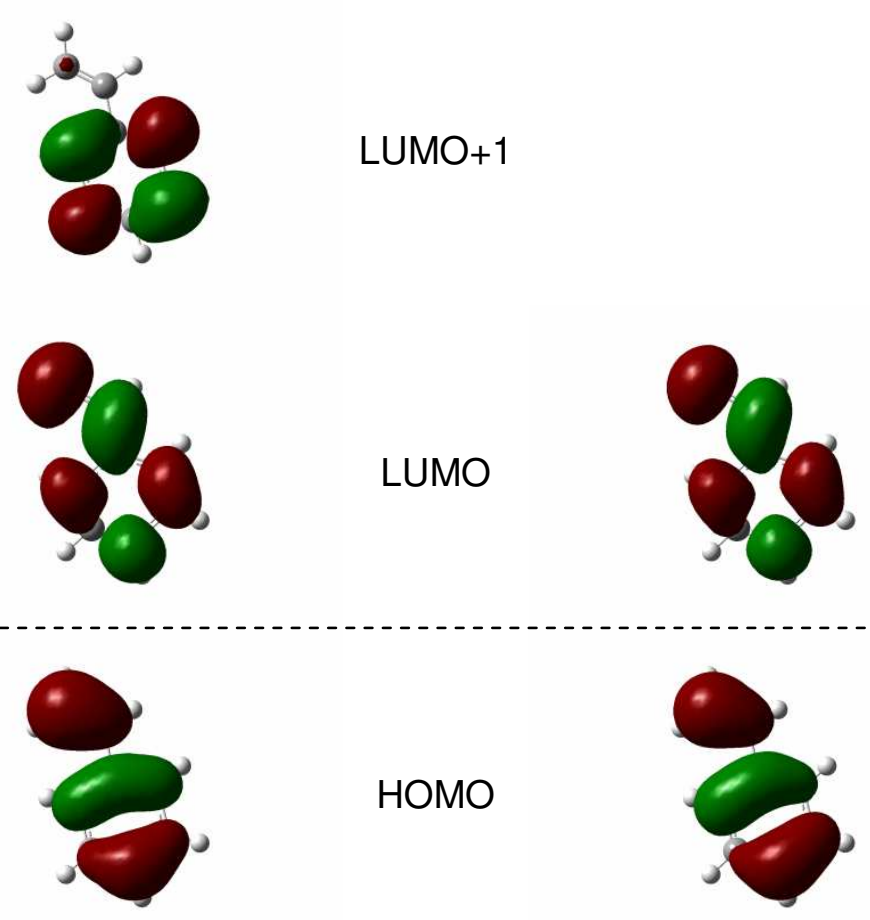

HOMO
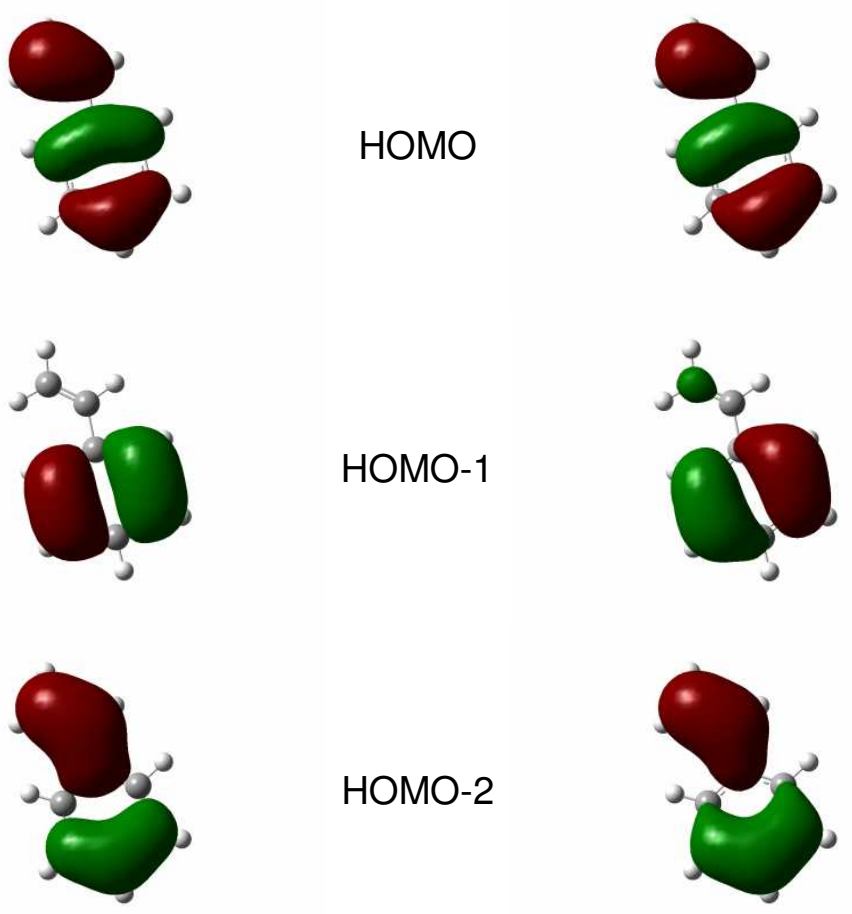

HOMO-2

HOMO-1

neutral

cation

Figure 2 
Figure 3
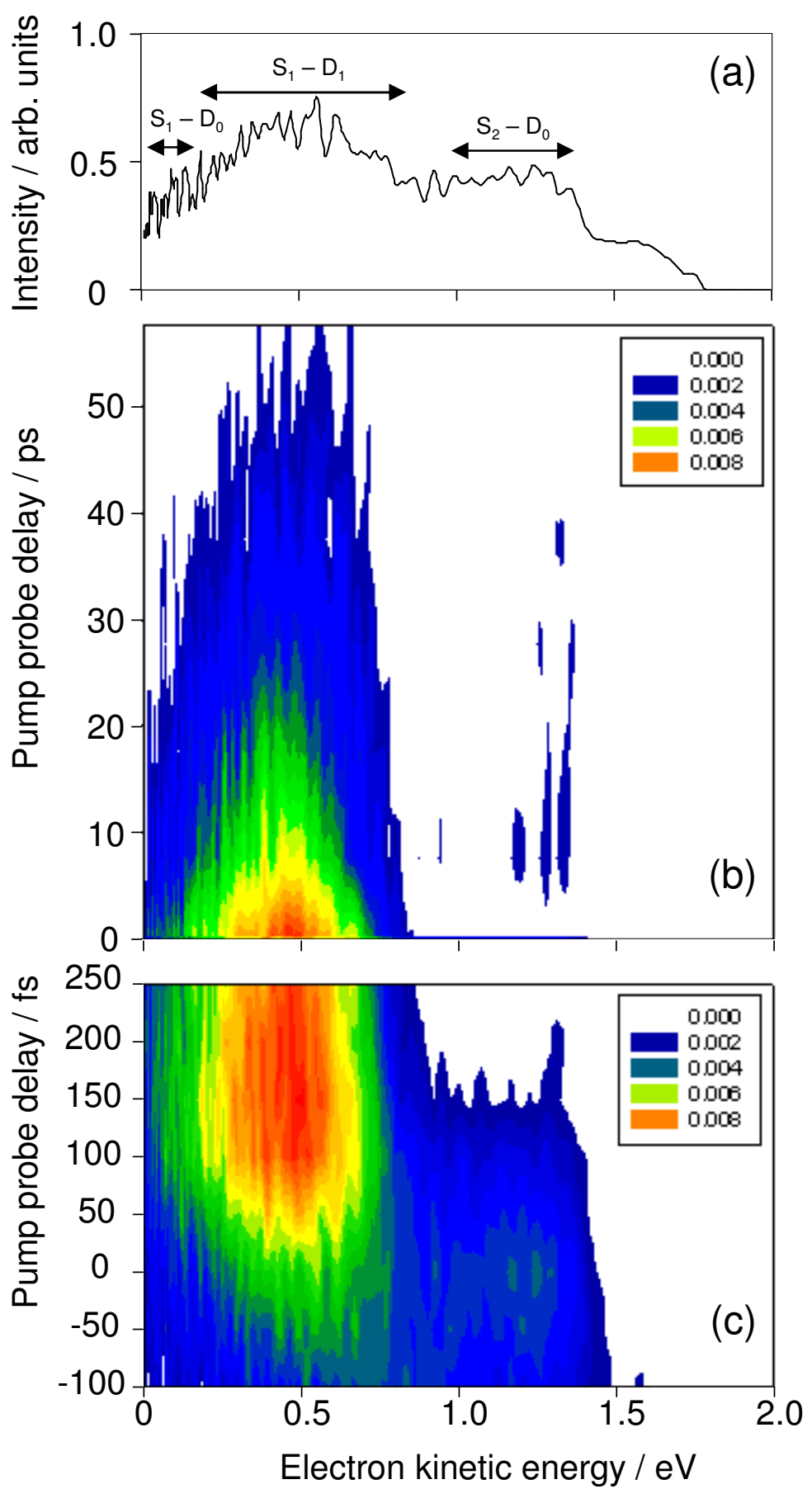

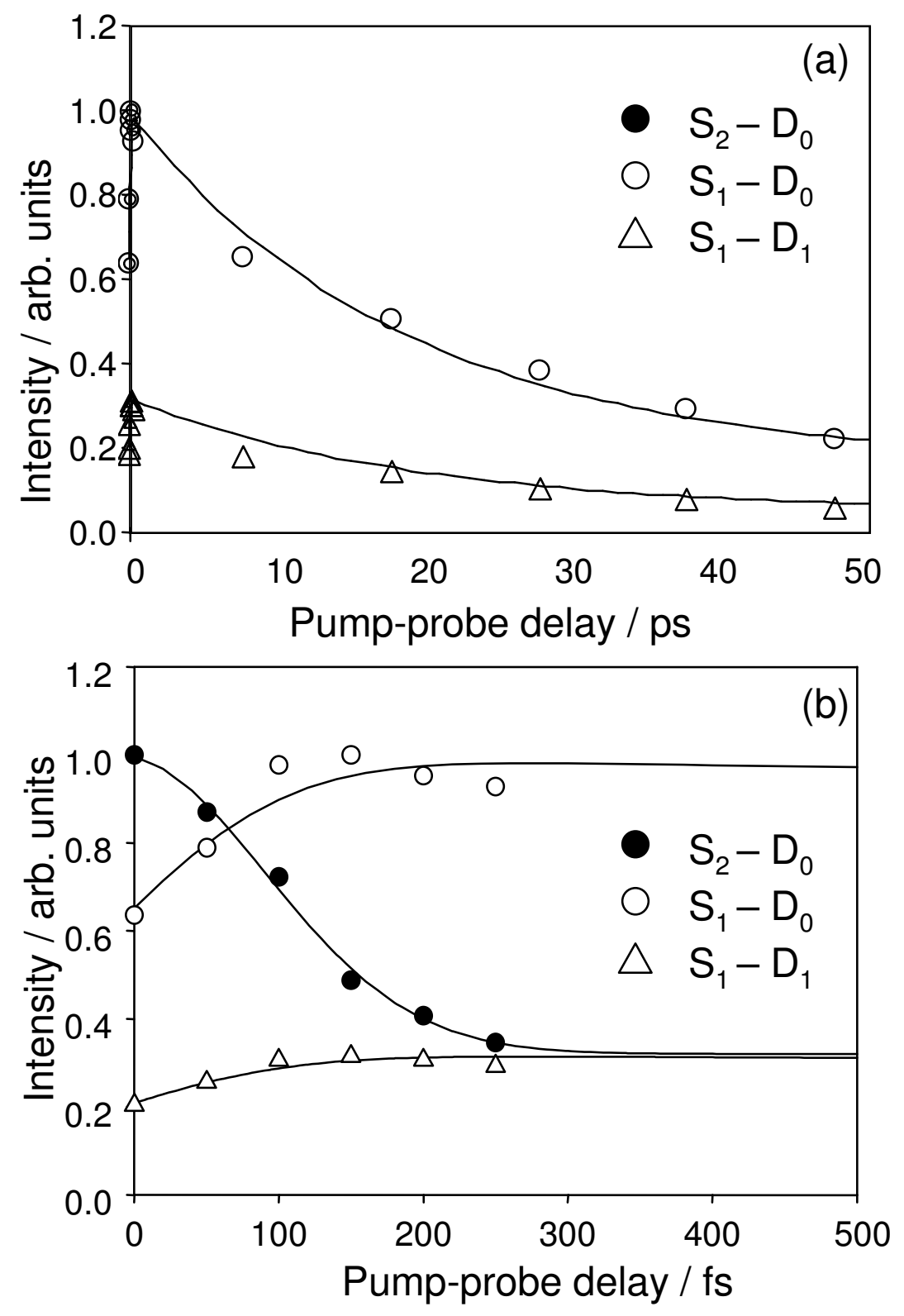

Figure 4 


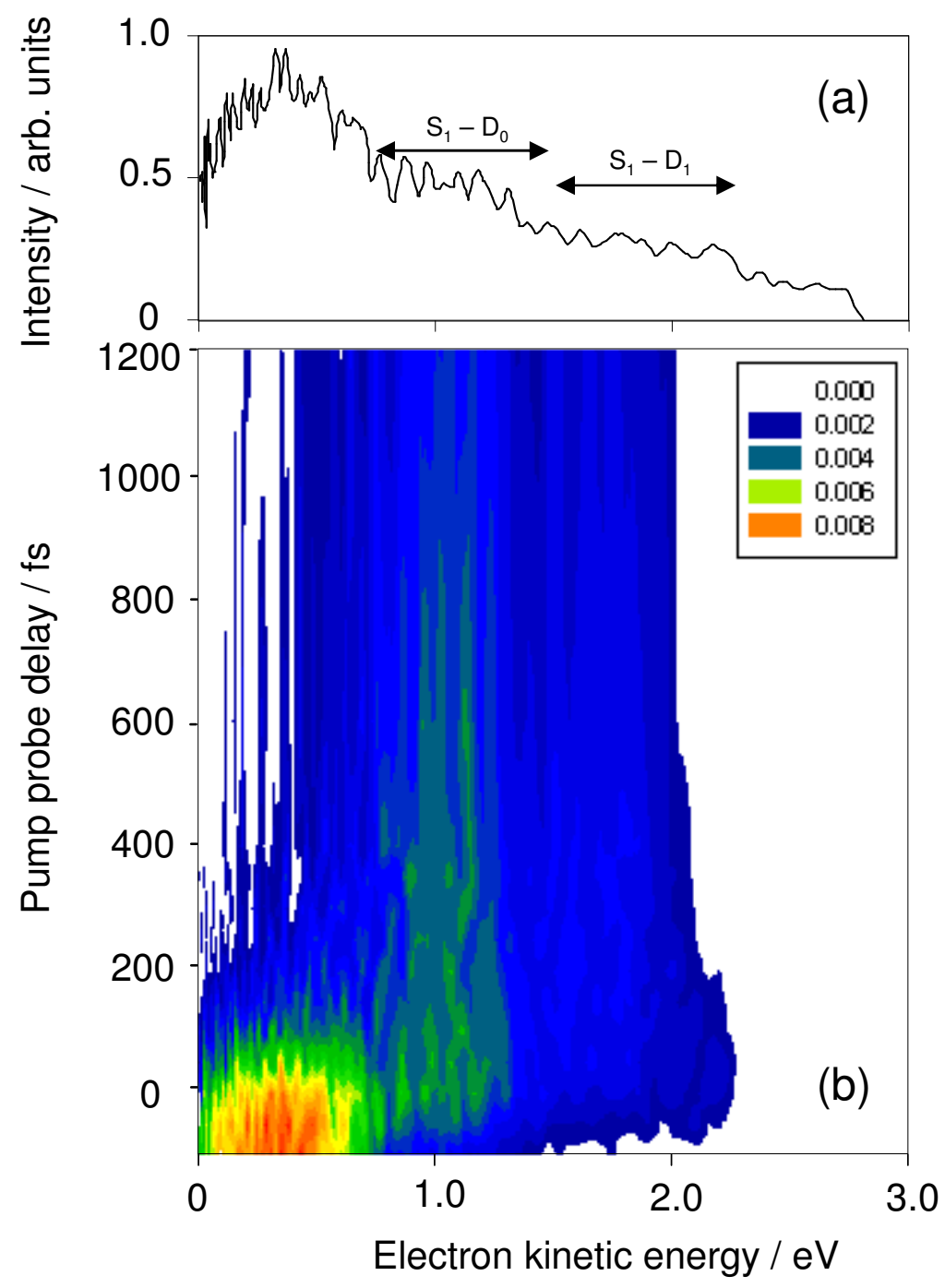

Figure 5 

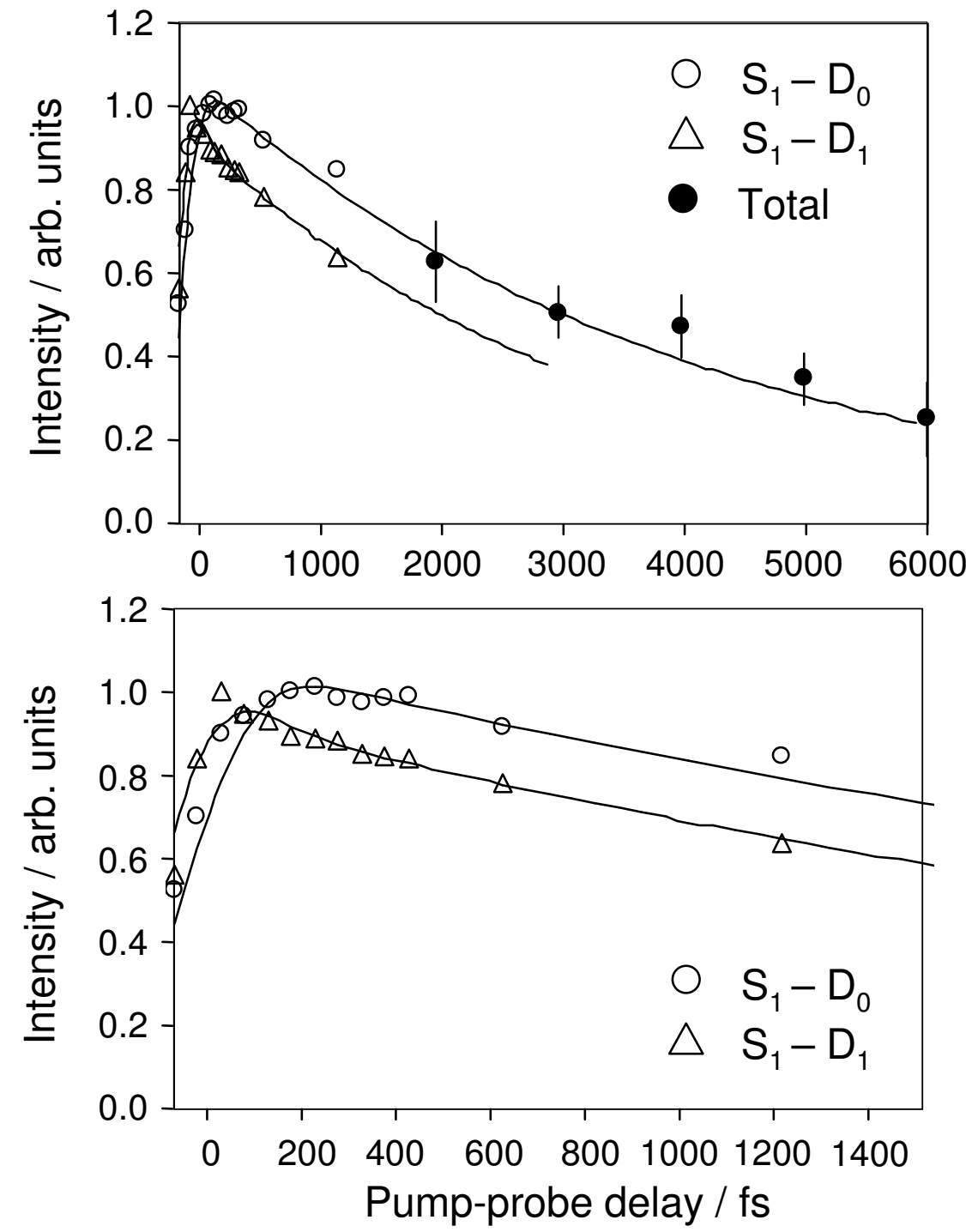

Figure 6 


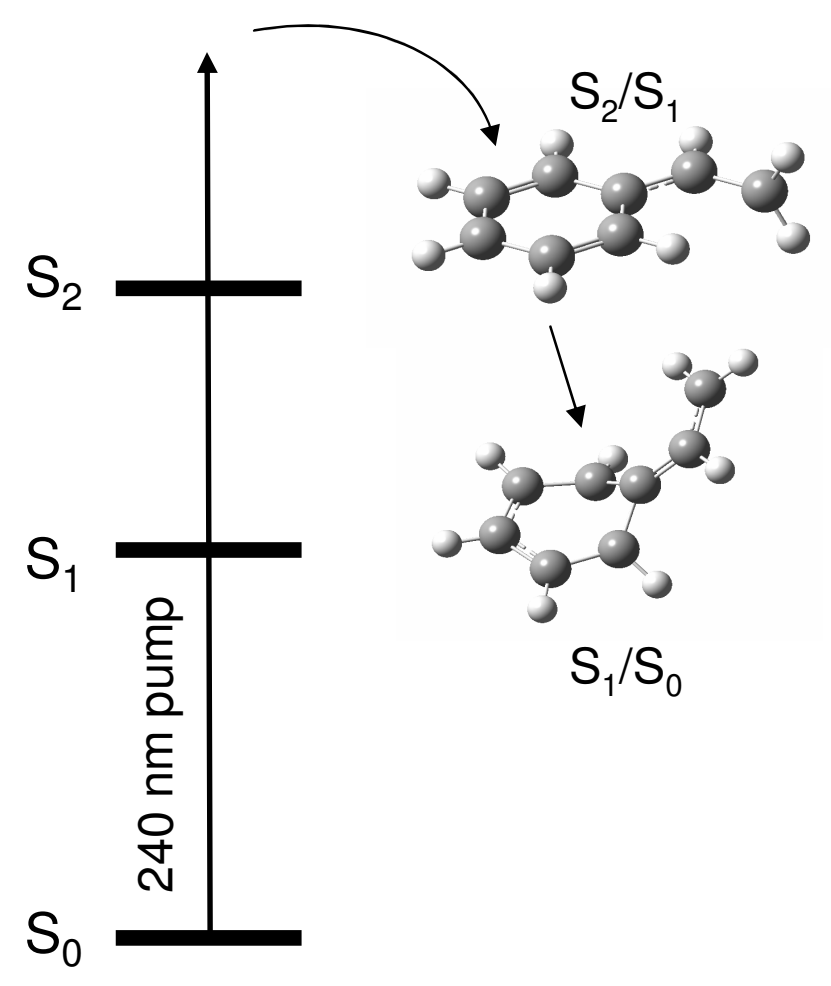

Figure 7 